\title{
LBNL-48851
}

\section{Observations of Microstructural Coarsening in Micro Flip-Chip Solder Joints}

\author{
Monica M. Barney ${ }^{*}{ }^{\dagger}$ and John W. Morris, Jr. ${ }^{\dagger}$
}

*Chemistry and Materials Science Directorate, University of California, Lawrence Livermore National Laboratory P.O. Box 808, Livermore, CA 94550, U.S.A.

$†$ Materials Sciences Division, Ernest Orlando Lawrence Berkeley National Laboratory, One Cyclotron Road, MS 66-200, Berkeley, CA 94720, U.S.A.

\begin{abstract}
Coarsening of solder microstructures dramatically affects fatigue lifetimes. This paper presents a study of microstructural evolution due to thermal cycling and aging of small solder joints. The lead-tin solder joints in this study have a height of $55 \pm 5 \mu \mathrm{m}$ and a tin content of $65-70 \mathrm{wt} \%$, with a degenerate eutectic microstructure. The joint microstructure coarsens more rapidly during aging at $160^{\circ} \mathrm{C}$ than cycling from $0^{\circ} \mathrm{C}-160^{\circ} \mathrm{C}$. No coarsened bands are observed. The cycling data scales with standard coarsening equations, while the aging data fits to an enhanced trend. The joints experiencing $2.8 \%$ strain during cycling fail by 1000 cycles.
\end{abstract}

Key Words: Flip-chip, small solder joints, coarsening, thermal fatigue 


\section{INTRODUCTION}

Flip-chip bonding is a method of electrically and mechanically attaching an integrated circuit (IC) to a substrate, usually with eutectic $63 / 37 \mathrm{wt} \% \mathrm{Sn} / \mathrm{Pb}$ solder. In this process, the IC is flipped face down, and the solder on the pads of the IC is bonded to corresponding pads on the substrate ${ }^{1}$. The two materials (the IC and the substrate) to which the solder is bonded invariably have much different thermal expansion coefficients. Therefore, when the device is subjected to thermal fluctuations, the solder joints undergo a large shear displacement with every cycle. In most cases, the solder is the most compliant material within a package, and it is assumed that the solder takes up all the strain produced by the thermal cycles ${ }^{2}$. While flip-chip bonding is not a new technique, it is recently being applied to the consumer electronic packages using Pb-Sn eutectic solder bonds and organic packages. The ever increasing demand for higher density packaging and size reduction are being met with the flip-chip design by decreasing the pad size and pitch of the solder joints. The joints that result from this type of design are typically $100 \mu \mathrm{m}$ or less in height and are termed microjoints ${ }^{3}$. With this design, a much smaller volume of solder is bearing a potentially higher strain. Because the solder joints in the flip-chip surface-mount design are the sole electrical and mechanical attachments, the reliability of the solder joints in the newer flip-chip design is even more critical.

It is difficult to assess the reliability of these smaller joints, because data available is primarily on larger joints with more compliant configurations and may not be applicable for this size. In addition, the initial microstructure of the smaller joints is expected to be different from the large joints due to the larger undercooling during the rapid solidification ${ }^{4}$. Under these conditions, non-lamellar structures of primary $\mathrm{Pb}$-rich dendrites supersaturated with $\mathrm{Sn}$ form within a $\mathrm{Sn}$-rich matrix containing randomly dispersed $\mathrm{Pb}$-rich particles. Upon further cooling, the Sn-rich phase precipitates out of the $\mathrm{Pb}$-rich dendrites as the solubility decreases with temperature. It is not known how this microstructure will evolve with time.

It has been found that, upon accounting for coarsening in large joints, the estimated fatigue life was only $21 \%$ of that found without including a coarsening parameter ${ }^{5,6}$. Therefore, observation of the microstructure is essential to assess approximate fatigue lifetimes for the small joints by determining the coarsening trends and failure mechanisms. Of the few that have undertaken microstructural studies to observe coarsening and its role in failure mechanisms and reliability, the majority of these studies have been performed on relatively large solder joints that have a predominately eutectic microstructure. In these studies ${ }^{7,8}$ it was determined that, during thermal cycling, the joints undergo an inhomogeneous shear deformation that leads to recrystallization and coarsening concentrated in bands of maximum shear. A crack eventually forms in this coarsened band and propagates to failure. This failure mode is found in the larger joints nearly independent of testing condition, as long as strain and temperature are present ${ }^{9}$. Because both the initial microstructure and the stress and strain distributions are different, it is not known how temperature and deformation will affect the microstructure in the smaller joints. This study was undertaken to produce some observations along these lines. 


\section{EXPERIMENTAL DETAILS}

Test samples were made by standard lithographic techniques to investigate the effect of different thermal treatments on small solder joints. Each sample consists of a quartz substrate, metallization layers, solder joints, and a silicon substrate. A schematic of the cross section of the samples after joining is shown in Figure 1. The pattern exposed onto the photoresist consists of two sets of three rows of 21 circles that were $100 \mu \mathrm{m}$ in diameter with a $250 \mu \mathrm{m}$ pitch. Solder was electroplated to the desired height of $55 \pm 5 \mu \mathrm{m}$. Because of the variability of the resultant composition of the plated metal inherent to this deposition technique, the actual composition of the solder was not precisely eutectic, but slightly Sn-rich (65-70\% Sn). This was determined by both quantitative stereology as well as with energy dispersive X-ray analysis.

Eutectic $\mathrm{Pb} / \mathrm{Sn}$ was chosen because its thermal fatigue response has been investigated previously on larger joints, which allows for a comparison with the smaller joints in this study. In an actual consumer package, this type of solder would be bonded to an organic substrate. This type of sample was made, but they failed after only a short time with thermal cycling. Easing the severity of the testing conditions must be done in order to investigate these samples. Quartz/silicon samples were successfully fabricated and tested to produce some initial observations of small joint behavior. The initial microstructures of both types of samples are nearly the same, so the observations presented here are of general interest.

After plating, each wafer was dipped for 15 seconds into glycerol that was heated to $215_{i} \mathrm{C}$ to melt the solder bumps. Each wafer was then diced into $7 \mathrm{~mm}$ by $11 \mathrm{~mm}$ pieces. One piece from a quartz wafer and one piece from a silicon wafer were bonded together by optically aligning the plated solder on each substrate and compression bonding them using an M- 8 Series Flip-Chip Bonder. Rosin flux was applied before compression bonding to ensure the removal of the oxide layer during subsequent melting. After compression bonding, all samples were reflowed under nitrogen with a preheat of $170^{\circ} \mathrm{C}$ for 30 seconds, followed by a reflow step lasting 180 seconds that peaked at $290^{\circ} \mathrm{C}$. The joints were then cooled at a rate of approximately $1^{\circ} \mathrm{C} /$ second.

To perform the thermal fatigue test, one group of samples was placed in a programmable furnace that cycled between 0$160_{i} \mathrm{C}$. The thermal cycle included five minute hold times at both zero and $160_{i} \mathrm{C}$. Heating from $0-160_{i} \mathrm{C}$ took approximately five minutes and cooling from $160-0_{i} \mathrm{C}$ took about 9 min, resulting in a 24 minute cycle. This test condition resulted in a strain of $2.8 \%$ per cycle. A second set of samples was isothermally aged in a low temperature furnace at $160_{i} \mathrm{C}$ for equivalent amounts of time as the cycled samples. An equivalent amount of time can be defined as the time of one cycle $\left(\mathrm{t}_{\mathrm{eq}}=24\right.$ $\mathrm{min} / \mathrm{cycle})$ or time at $160_{i} \mathrm{C}\left(\mathrm{t}_{\mathrm{eq}}=5 \mathrm{~min} / \mathrm{cycle}\right)$, and both definitions were employed in this study to evaluate the data. 
To quantify the microstructures, quantitative stereology was performed by counting the number of phase boundaries a

test line intersects, indiscriminant of phase type. This $\mathrm{Sn} / \mathrm{Pb}$ phase size, $\mathrm{d}_{\mathrm{Sn} / \mathrm{Pb}}$, was tabulated and used as a parameter to

characterize the microstructure and microstructural evolution of each sample. It was indicated in another study ${ }^{10}$ that this parameter is a good indicator of coarsening.

\section{DISCUSSION}

An example of the initial microstructure is shown in Figure 2. All micrographs were obtained with an optical microscope. As anticipated, a globular, degenerate eutectic microstructure is observed in all of the samples used in this study. Neither the lamellar eutectic microstructure nor any colony boundaries were seen. The microstructure consists of $\mathrm{Pb}$-rich dendrites with Sn-rich particles residing in a Sn-rich matrix with isolated Pb-rich particles. This microstructure is observed in these joints even if the cooling rate is substantially reduced, indicating that the amount of undercooling, not the cooling rate, is the critical factor in determining the initial microstructure for small volume solder joints. The organic/silicon samples also produced this type of initial microstructure. Most joints contained voids that varied greatly in size, percentage of joint area, and location within the joint.

Isothermal aging uniformly coarsens the microstructure of these small joints (Figure 3), as is expected at high temperatures with no strain. The Sn-rich particle size is seen to increase within the $\mathrm{Pb}$-rich dendrites. The number of isolated $\mathrm{Pb}$-rich particles is seen to decrease as they diffuse toward the dendrites. Thermal cycling did not produce the coarsened shear bands that are seen in larger joints under these conditions. Instead, the microstructure coarsened uniformly (see Figure 4) with minor phase diffusing to the parent phase, as seen in the aged microstructures. The results of the quantitative stereology (Figure 5) also show greater coarsening in the aged samples. This result is obtained for both definitions of equivalent time. The trend of $t_{\mathrm{eq}}=5 \mathrm{~min} / \mathrm{cycle}$ is closer to the cycling data, but is still above it. This coarsening trend is the exact opposite of what has been reported before for larger joints ${ }^{8}$. The cycling data scaled with the general form of a cubic coarsening law derived for standard diffusion behavior ${ }^{11}$, which the aging data did not (Figure 6). The aging data scaled with the general form of an enhanced coarsening law $^{11}$ (Figure 7). The two equations mentioned are shown below.

$$
\text { Cubic Coarsening: } \mathrm{d}^{3}-\mathrm{d}_{\mathrm{o}}{ }^{3}=\mathrm{B}\left(\gamma \Omega \mathrm{C}_{\mathrm{o}} \mathrm{D}_{\mathrm{v}} / \mathrm{RT}\right) \mathrm{t}
$$

where $r$ is the particle radius, B is a constant, $\gamma$ is the interfacial free energy per unit area, $\Omega$ is the molar volume of the particles, $C_{o}$ is the solute concentration in equilibrium, $D_{v}$ is the coefficient of volume diffusion, and RT has its standard meaning.

Enhanced Coarsening: $\mathrm{d}^{4}-\mathrm{d}_{\mathrm{o}}{ }^{4}=\mathrm{K}(\mathrm{d} \varepsilon / \mathrm{dt})\left[\mathrm{t}-\tau_{\mathrm{c}}+\tau_{\mathrm{c}} \exp \left(-\mathrm{t} / \tau_{\mathrm{c}}\right)\right]$ 
where $\mathrm{d}$ is the particle diameter, $\mathrm{K}$ is a constant, $\mathrm{d} \varepsilon / \mathrm{dt}$ is the strain rate, and $\tau_{\mathrm{c}}$ is the relaxation time of excess vacancies.

The data from this study was fit with just the general form of these equations as shown below:

General form of Cubic Coarsening: $\left(\mathrm{d}_{\mathrm{Sn} / \mathrm{Pb}}\right)^{3}-\left(\mathrm{d}_{\mathrm{Sn} / \mathrm{Pb}}\right)_{\mathrm{o}}{ }^{3} \propto \mathrm{t}$

General form of Enhanced Coarsening: $\left(\mathrm{d}_{\mathrm{Sn} / \mathrm{Pb}}\right)^{4}-\left(\mathrm{d}_{\mathrm{Sn} / \mathrm{Pb}}\right)_{0}^{4} \propto[\mathrm{t}-\exp (-\mathrm{t})]$

It is not yet known the exact mechanism causes this opposite trend. It has not been determined from these measurements whether the cycling is somehow slowing coarsening or if aging is enhancing coarsening. During thermal cycling, the minor phase under goes dissolution and reprecipitation with each thermal cycle due to the change in solubility of $\mathrm{Sn}$ in $\mathrm{Pb}$. This could be slowing the coarsening, since, during aging, the Sn-rich particles grow and coalesce without interruption. The equation with which the aging data scales was derived assuming that enhanced coarsening is caused by an excess vacancy concentration created by strain. Here, the excess vacancies could be coming from the relatively high temperature of these tests instead of from strain, since the tests in the present study were run at higher temperatures and lower strains than other studies. More test must be done on these samples to determine if any of these possibilities are occurring.

The thermal fatigue specimens failed by 1000 cycles under $2.8 \%$ strain. These are shorter lifetimes than a study that used higher strain and lower temperatures ${ }^{9}$, but longer lifetimes than some other studies on large and small joints ${ }^{10,11}$. As always, it is difficult to compare to other studies directly, but it appears that this lifetime is not unusually long. The failure mode of the small joints in this study was different than in studies on larger joints. In larger joints, the failure mode included the formation of a coarsened shear band in the solder near the solder/substrate interface. This band propagated across the joint in the solder parallel to the interface. This pattern was observed in larger joints as long as strain and high temperature were present (e.g., thermal fatigue, isothermal fatigue at high temperature, and creep), even when the initial microstructure was non-lamellar ${ }^{9}$. Fatigue cracks would initiate and propagate through these bands. In the small joints, fatigue cracks also initiated in the solder near the solder/substrate interface and propagated through the solder parallel to the interface. They would deflect away from this path only if a void was present. However, no coarsened bands were observed to form even upon failure. It is not clear at this time why there is this difference in failure mode.

\section{CONCLUSIONS}

Due to large undercoolings, initial microstructures for near-eutectic $\mathrm{Sn} / \mathrm{Pb}$ joints consist of a degenerate eutectic morphology. The coarsening during aging was more rapid than during thermal cycling but tapered off at a faster rate. The microstructural coarsening during cycling appeared to follow traditional coarsening laws, while the coarsening during aging scaled with an enhanced coarsening relation. The enhanced coarsening behavior during aging is possibly due to excess 
vacancies from the relatively high temperatures. However, the exact mechanism for the difference in coarsening behavior is not known at this time. It is critical to determine these mechanisms since coarsening strongly affects fatigue lifetime. This is a more realistic undertaking now that the thermal behavior of small solder joints has been observed.

\section{ACKNOLEDGMENTS}

The authors would like to thank Dr. Chris Keller for helpful discussions as well as assistance in fabrication and use of equipment. The authors would also like to thank Mr. Rudy Bartolo for his electroplating work. All photolithography work was performed in the Microfabrication Facility at the University of California, Berkeley. This work was supported by the Director, Office of Energy Research, Office of Basic Energy Sciences, Materials Science Division of the U.S. Department of Energy under Contract No. DE-AC03-76SF00098.

\section{REFERNCES}

1. E. J. Vardaman, I. Yee, and R. Crowley, Worldwide Developments in Flip-Chip Interconnect, (Electronics Industries Association, 1994)

2. H. Frost, in Solder Joint Reliability, Theory and Applications, edited by J. H. Lau, (Van Nostrand Reinhold, 1991)

3. J.H. Lau, Flip-Chip Technologies (McGraw-Hill, 1996)

4. N. R. Green, J. A. Charles, and G. C. Smith, Materials Science and Technology, 10, 977 (1994)

5. P. Hacke, A. F. Sprecher, and H. Conrad, Journal of Electronic Packaging, 115, 153 (1993)

6. L. R. Fox and S. Knecht, IEEE Transactions on Components, Hybrids, and Manufacturing Technology, 13(2), (1990)

7. J. W. Morris, Jr., D. Tribula, T. S. E. Summers, and D. Grivas, in Solder Joint Reliability, Theory and Applications, edited by J. H. Lau (Van Nostrand Reinhold, 1991)

8. P. L. Hacke, A. F. Sprecher, and H. Conrad, Journal of Electronic Materials, 26 (7) (1997)

9. Z. Mei and J. W. Morris, Jr., Journal of Electronic Packaging, 114, 744 (1992)

10. P. Hacke, A. F. Sprecher, and H. Conrad, Journal of Electronic Packaging, 118, 49 (1996)

11. O. N. Senkov and M. M. Myshlyaev, Acta Metall., 34 (1), 97 (1986) 


\section{Quartz}

\section{Silicon}

Figure 1: Sample Schematic in Cross-section after reflow 

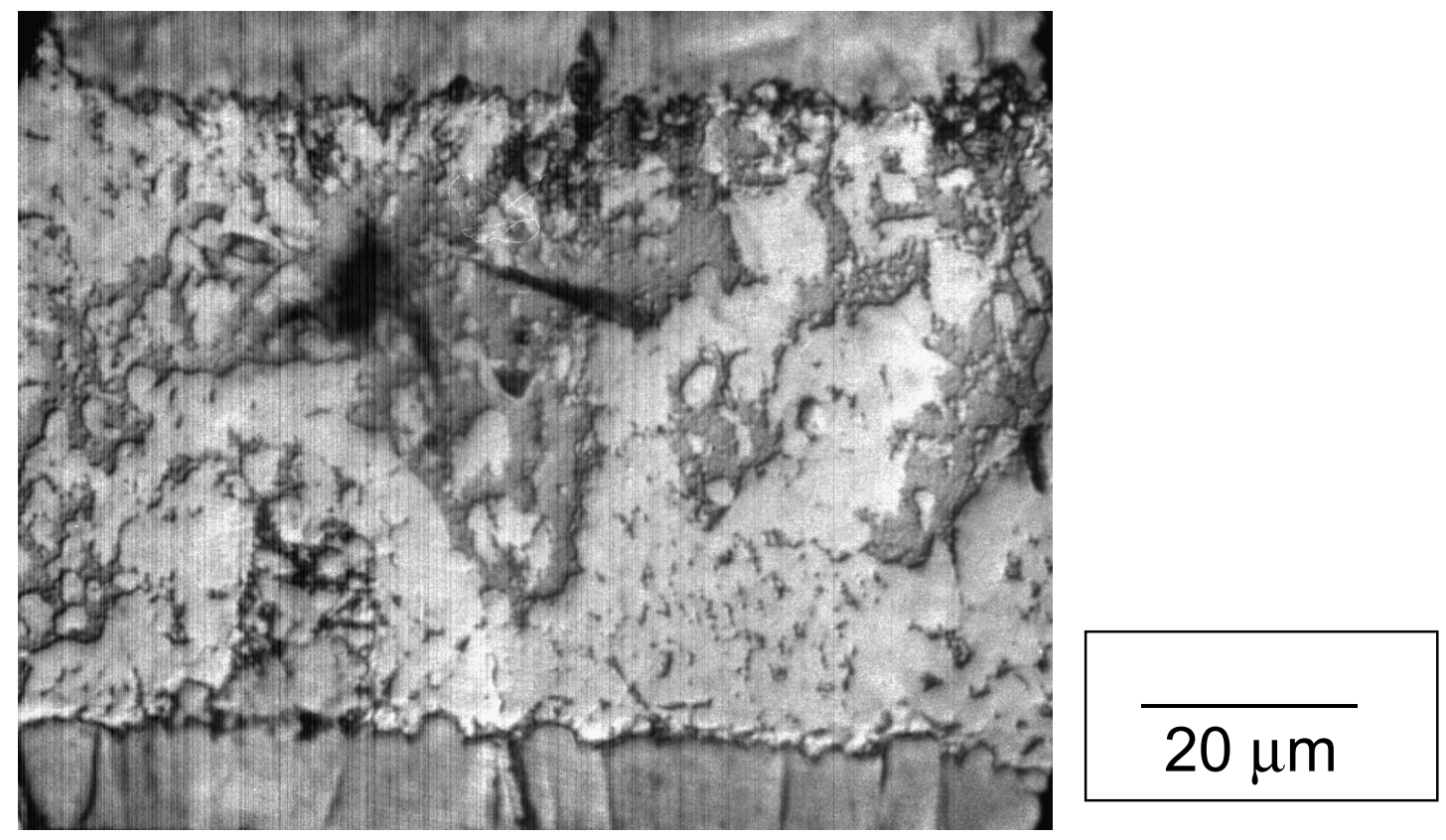

Figure 2: Initial Joint Microstructure 

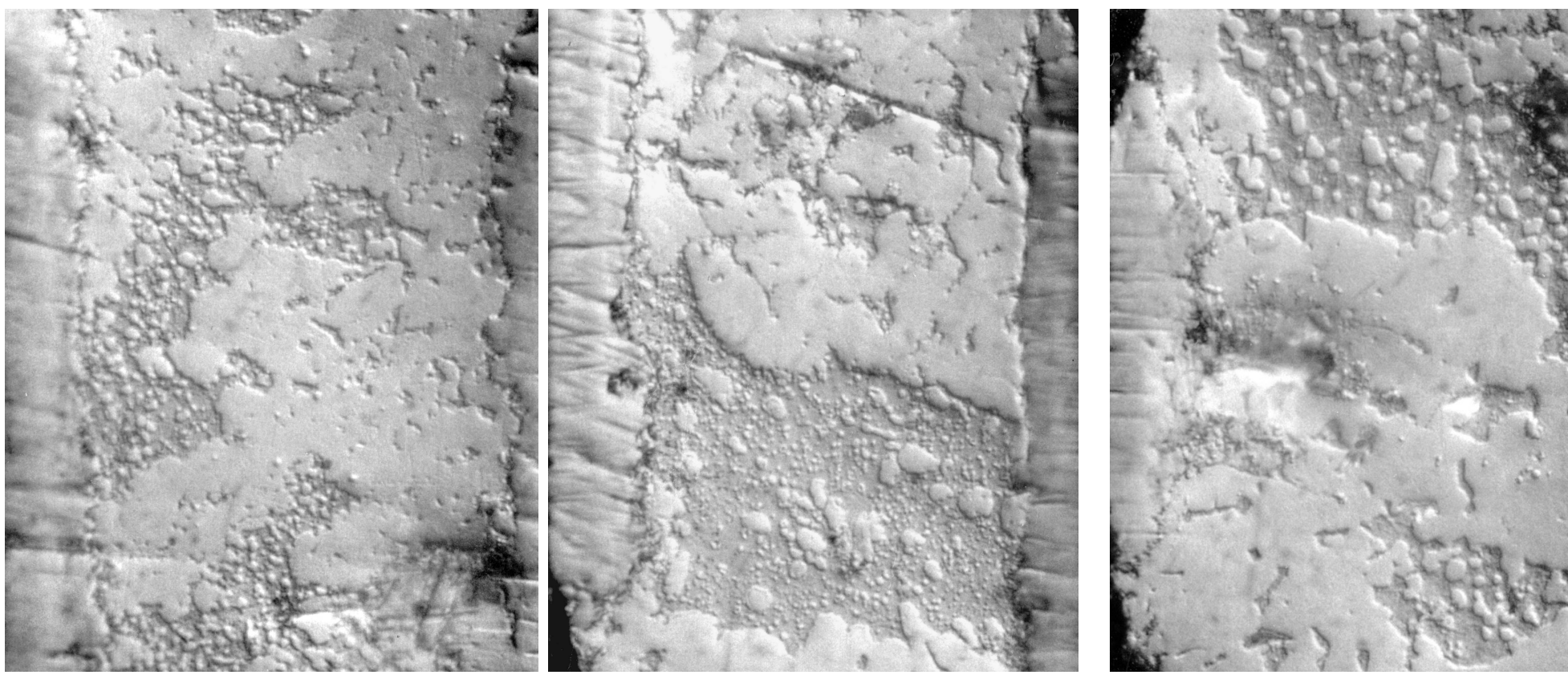

\section{0 minutes}

2500 minutes

50000 minutes

Figure 3: Microstructures of Aged Samples

$25 \mu \mathrm{m}$ 


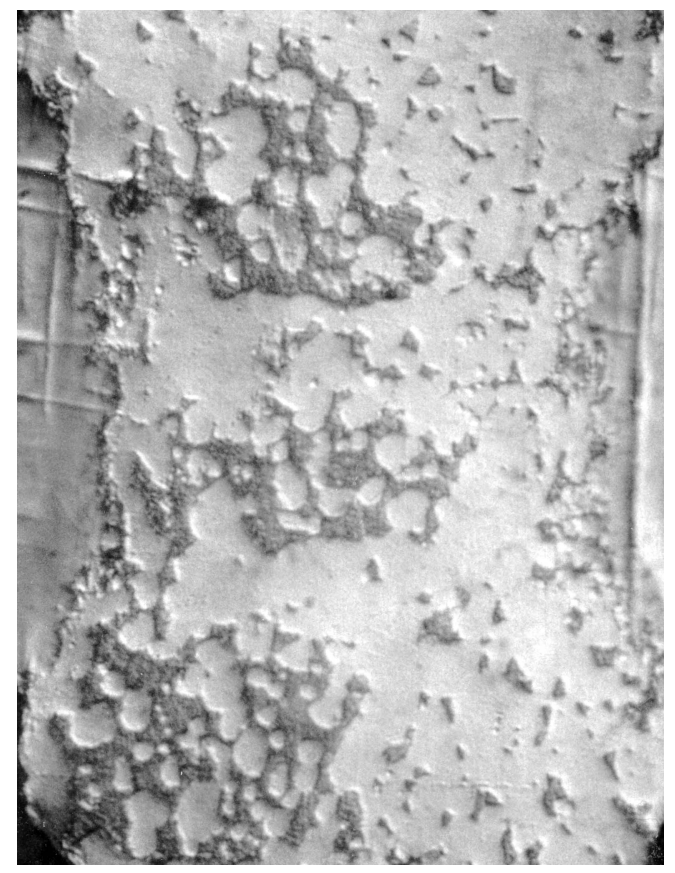

50 cycles
(1200 minutes)

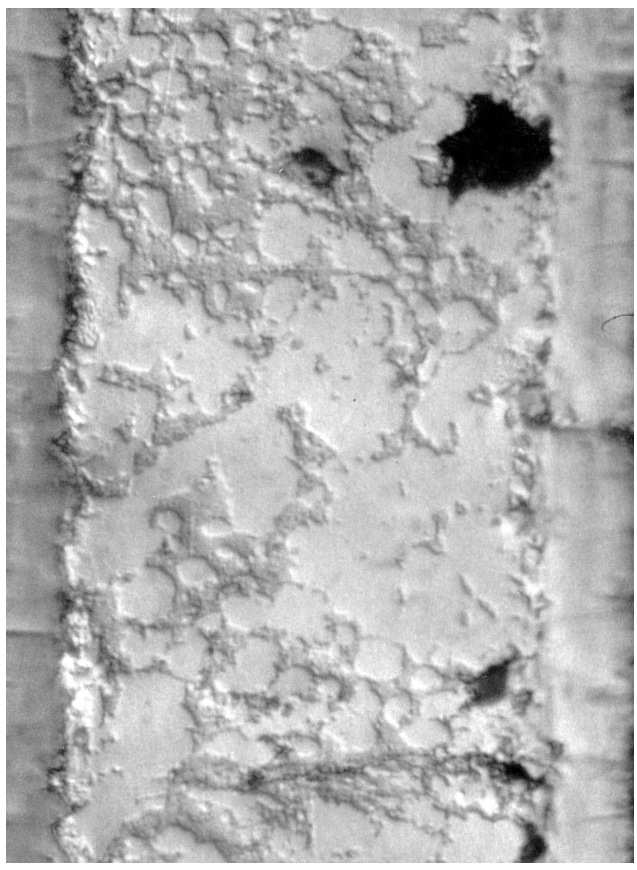

250 cycles (6000 minutes)

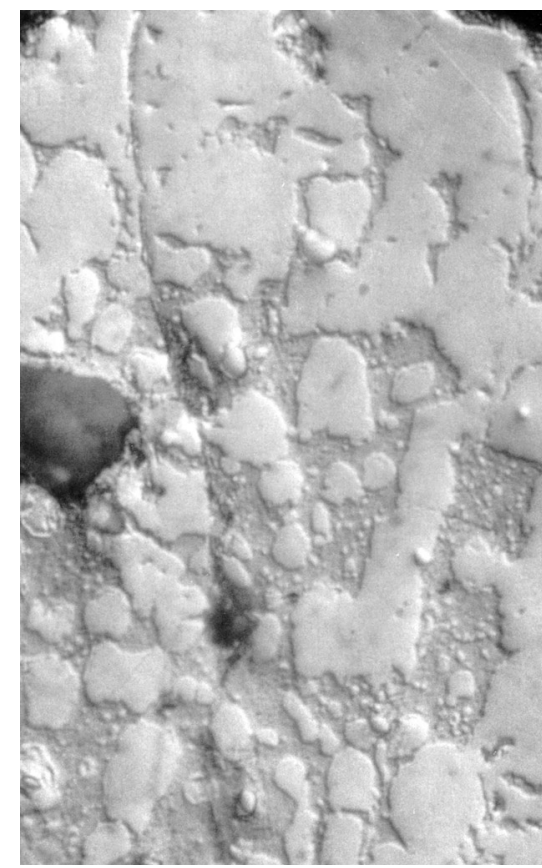

500 cycles
(12000 minutes 


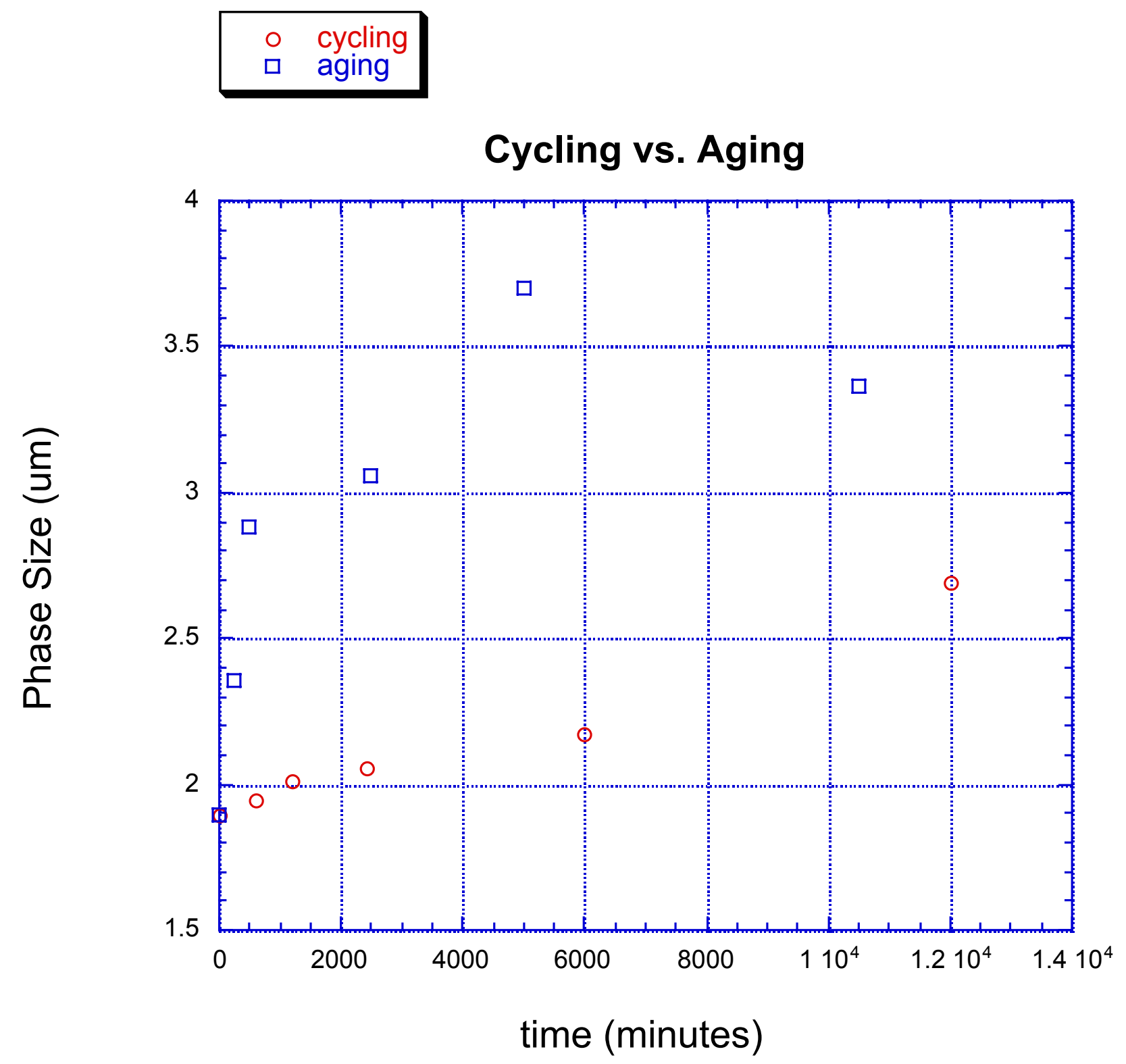

Figure 5: Cycling vs. Aging data 


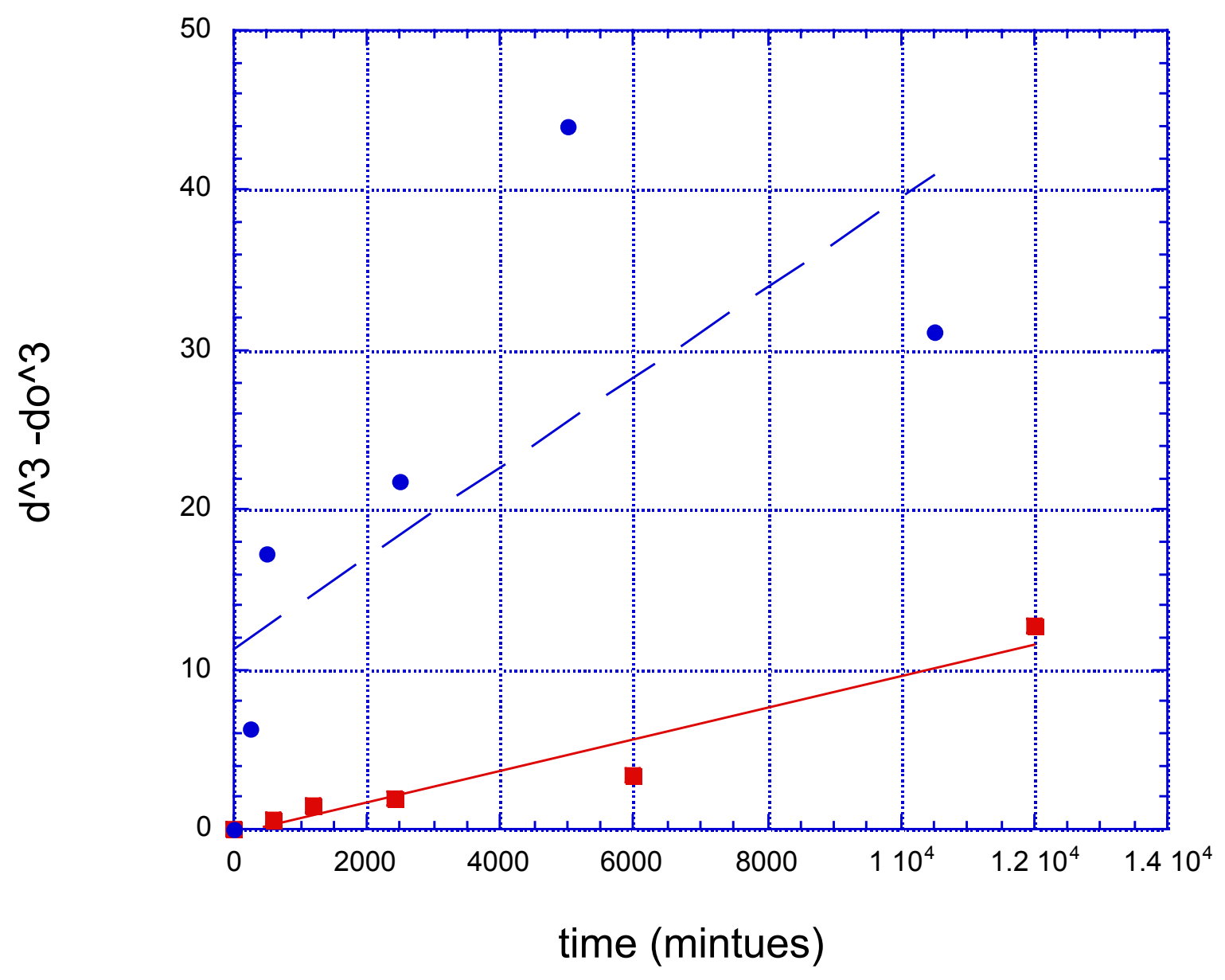

Figure 6: Plot of the standard cubic coarsening law (Eq. 1). The cycling data (squares) fits this plot well, but the aging data (circles) does not. 


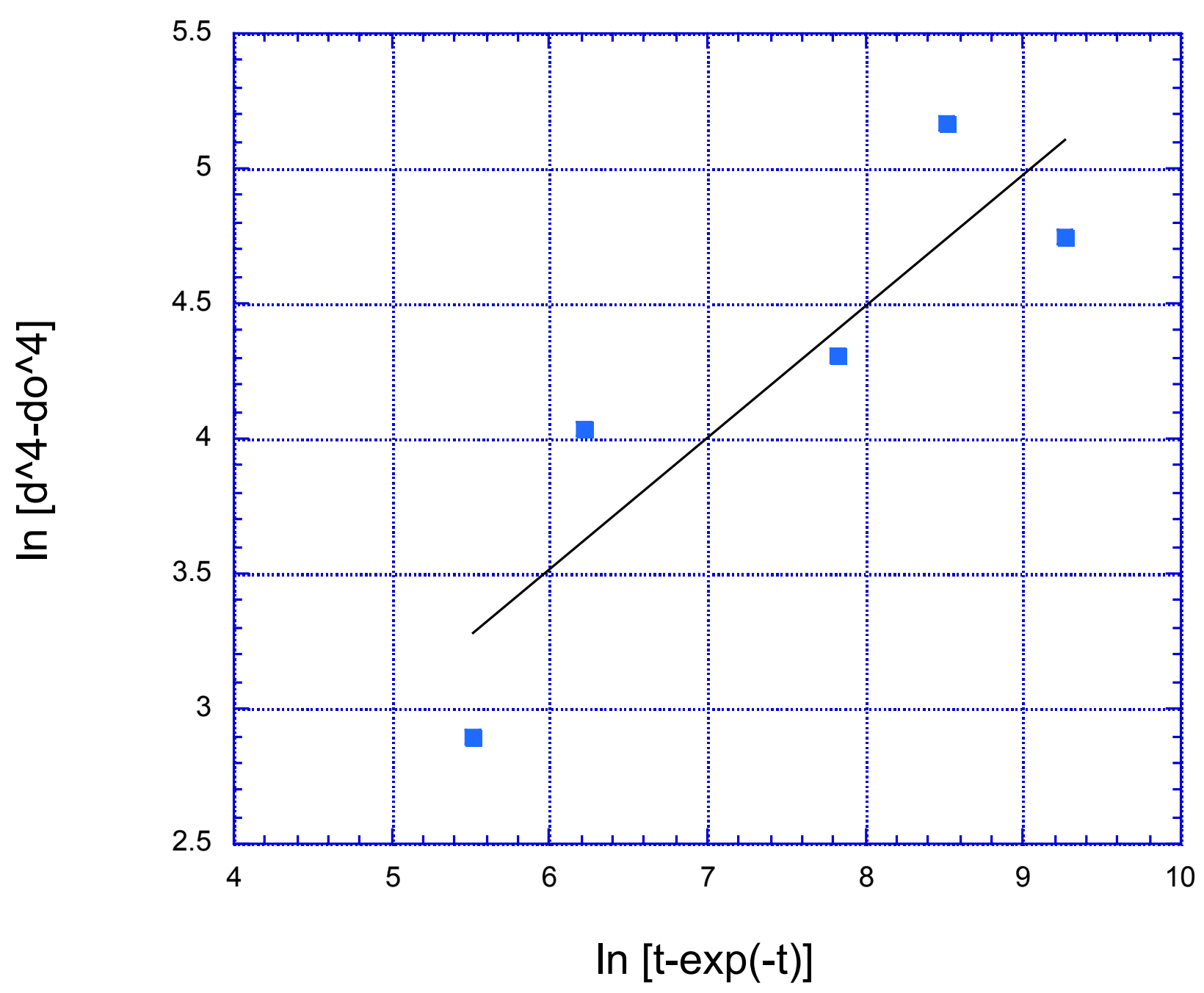

Figure 7: Plot of the aged data fit to an enhanced coarsening law (Eq. 2) 